Dynamic Estimation of the Sound-Speed Profile from Broadband Acoustic Measurements

O. Carriere, J. Hermand, M. Meyer, J. V. Candy

June 13, 2007

IEEE OCEANS '07 Aberdeen Scotland Aberdeen, United Kingdom June 18, 2007 through June 21, 2007 
This document was prepared as an account of work sponsored by an agency of the United States government. Neither the United States government nor Lawrence Livermore National Security, LLC, nor any of their employees makes any warranty, expressed or implied, or assumes any legal liability or responsibility for the accuracy, completeness, or usefulness of any information, apparatus, product, or process disclosed, or represents that its use would not infringe privately owned rights. Reference herein to any specific commercial product, process, or service by trade name, trademark, manufacturer, or otherwise does not necessarily constitute or imply its endorsement, recommendation, or favoring by the United States government or Lawrence Livermore National Security, LLC. The views and opinions of authors expressed herein do not necessarily state or reflect those of the United States government or Lawrence Livermore National Security, LLC, and shall not be used for advertising or product endorsement purposes. 


\title{
Dynamic Estimation of the Sound-Speed Profile from Broadband Acoustic Measurements
}

\author{
Olivier Carrière*, Jean-Pierre Hermand*, Matthias Meyer* and James V. Candy ${ }^{\dagger}$ \\ *Environmental Hydroacoustics Lab., Dept. of Optics and Acoustics \\ Université libre de Bruxelles (U.L.B.) \\ Avenue, Franklin D. Roosevelt, 50 - CP 194/05 \\ B-1050 Brussels, Belgium \\ Phone: $+32(0) 26736527$ \\ Email: jhermand@ulb.ac.be \\ †University of California, Santa Barbara \\ \& \\ Lawrence Livermore National Laboratory \\ P. O. Box 808 \\ Livermore, CA 94551 \\ USA
}

\begin{abstract}
Global search and more recently adjoint-based inversion methods used in ocean acoustics showed their effectiveness in the estimation of the sound-speed profile (SSP) in water columns of several environments. In the framework of the European Seas Observatory Network (ESONET) an essential part of the research and technology focuses on continuous and long term observations to characterize dynamic ocean processes and monitor the global state of the ocean. Therefore, the development of high performançe integrated tools for acoustic inversion is one of the attractive components in this network. For the purpose of efficient data assimilation this paper investigates sequential methods that are able to update soundspeed profile parameters, typically the coefficients of empirical orthogonal functions (EOF), with respect to new incoming acoustic or hydrographic measurements and take into account the seafloor and sub-seafloor acoustic properties in a shallow water environment. A formulation using Kalman filters is suitable for a sequential treatment. This paper investigates the application of two different extensions of the Kalman filter, the extended Kalman filter and the more recent unscented Kalman filter for comparison.
\end{abstract}

Index Terms-ocean acoustic tomography, unscented Kalman filter, empirical orthogonal functions

\section{INTRODUCTION}

Global search and more recently adjoint-based inversion methods used in ocean acoustics showed their effectiveness in the estimation of geometric and (geo)acoustic parameters of several environments ([1]-[3] and many others). The soundspeed profile (SSP) is an important source of information in order to retrieve ocean properties (temperature, salinity,...). In the framework of the European Seas Observatory Network $\left(E_{S O N E T}{ }^{1}\right)$ an essential part of the research and technology focuses on continuous and long term observations to characterize dynamic ocean processes and monitor the global state of the ocean. Therefore, the development of high performance

\footnotetext{
${ }^{1}$ see website http://www.ifremer.fr/esonet/
}

integrated tools for acoustic inversion is one of the attractive components in this network.

Due to their stochastic nature, model-based processing approaches are well suited for data assimilation into numerical models, typically in meteorology and oceanography. The ocean-acoustic model and measurement system are placed into state-space form, allowing unknown signal and environmental parameters to be estimated simultaneously [4], [5]. A major advantage of the model-based approach is that there is no inherent limitation to the degree of sophistication of the models used, and therefore it can deal with different propagation models (normal-mode, parabolic equation, ...) and integrate oceanographic models.

Recently, some works have envisioned an integrated formulation of joint acoustic and oceanographic data assimilation, including both the acoustic and the oceanic variables in the state vector [6], [7]. For the purpose of efficient data assimilation this paper investigates sequential methods that are able to update sound-speed profile parameters, typically the coefficients of empirical orthogonal functions (EOF), with respect to new incoming acoustic or hydrographic measurements and take into account the seafloor and sub-seafloor acoustic properties in a shallow water environment.

This paper treats a shallow water case with broadband and full field measurements, as required by the use of sparse, vertical hydrophone arrays in a time-dispersive ocean waveguide [8].

Because variations in SSP perturb the pressure field, the model-based processor is constructed to extract time variations of the EOF coefficients. The Gauss-Markov representation enables the inclusion of stochastic phenomena such as measurement noise or modeling error into the processor. Modeling error terms can then represent the uncertainty on the evolution of the (slowly varying) SSP.

Non-linearity in the state-space formulation requires the use 
of suitable processors. The extended Kalman filter (EKF) is a sub-optimal processor that linearizes the dynamical equations around the current estimate of the state, under Gaussian assumptions. This paper proposes a design of both the EKF and an unscented Kalman filter (UKF). The UKF uses the same Gaussian assumptions than EKF, but distributions are represented using a minimal set of sample points to capture the statistics of the variables. The main advantage of UKF is the use of the true non-linear model to propagate the sample points [9].

The remainder of the paper is organized as follows. The state-space model is first introduced in Sec. 2. Section 3 then presents the essential principles of the UKF. Section 4 presents some results in a real environment. We conclude the paper in Sec. 5.

\section{GAUSS-MARKOV STATE-SPACE MODEL}

The use of empirical orthogonal function (EOF) is a good way to fully parameterize the sound-speed profile. EOF are basis functions that can be obtained from a database and are very efficient to reduce the number of data points to be estimated. The use of EOF has already proved its efficiency in sound-speed profile inversion problem ([10], [11] and many others).

The modeled sound-speed profile $c(z)$ can then be expressed in terms of a vector of coefficients $\underline{\alpha}$

$$
c(z)=\bar{c}(z)+\sum_{i=1}^{N} \alpha_{i} \delta c_{i}(z)
$$

where $\bar{c}(z)$ is the mean sound-speed profile computed on the historical data. The number $N$ of EOF is chosen so that, in terms of energy, more than $90 \%$ of the sound speed variation is described ( $N=3$ in our case).

Figure 1 shows the mean SSP and the first three EOF computed from the Yellow Shark (YS) database.

We propose to use EOF coefficients as the states $\alpha_{i}$ of our model. Indirect measurement of these states is given by the acoustic pressure field $p_{i}$ on a vertical array of $L$ hydrophones. The relation between the states and the acoustic pressure field is highly non-linear and is computed by the resolution of a normal-mode model. The state-space evolution model is given by

$$
\left(\begin{array}{c}
\alpha_{1}\left(t_{k}\right) \\
\vdots \\
\alpha_{N}\left(t_{k}\right)
\end{array}\right)=\mathcal{A}\left(\begin{array}{c}
\alpha_{1}\left(t_{k-1}\right) \\
\vdots \\
\alpha_{N}\left(t_{k-1}\right)
\end{array}\right)+\underline{w}\left(t_{k-1}\right)
$$

The measurement values are computed as

$$
\left(\begin{array}{c}
p_{1}\left(t_{k}\right) \\
\vdots \\
p_{L}\left(t_{k}\right)
\end{array}\right)=\mathcal{C}\left(\underline{\alpha}\left(t_{k}\right)\right)+\underline{v}\left(t_{k}\right)
$$

where $\mathcal{A}$ is the transition function, $\mathcal{C}$ is the (non-linear) measurement function that computes the acoustic pressure field

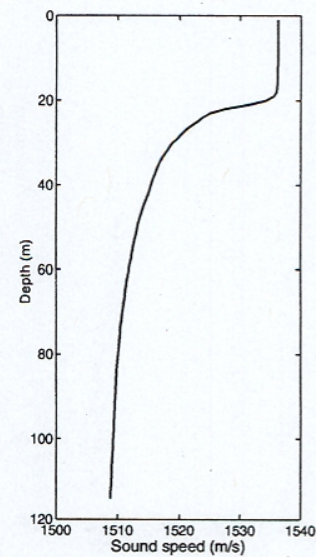

(a)

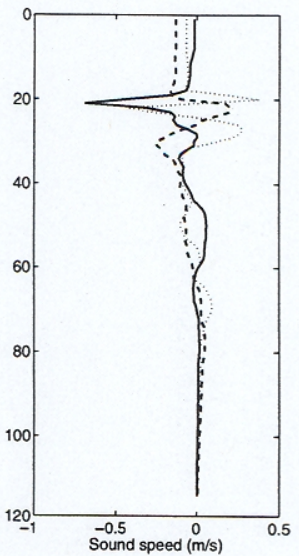

(b)
Figure 1. (a) Mean sound-speed profile of the database. (b) First three EOF computed on the database (solid line: EOF 1, dashed line: EOF 2, dotted line: EOF 3).

with respect to the state vector, and $\underline{w}\left(t_{k}\right)$ and $\underline{v}\left(t_{k}\right)$ are zeromean Gaussian random vectors (respectively of covariance $R_{w w}$ and $\left.R_{v v}\right)$. The components of $\boldsymbol{p}_{\boldsymbol{i}}\left(t_{k}\right)$ are the acoustic pressure amplitude at each frequency on the $i$ th hydrophone. Noise terms are assumed to be independent and uncorrelated (spatially and temporally).

For this paper, we would like to investigate the capability of the state-space formulation with Kalman filtering to to efficiently invert the SSP. We do not yet integrate suitable oceanic models, so the transition function is set to the identity matrix. However, the structure of the state-space model should integrate a more complex oceanic model, with augmenting simultaneously the state vector and the measurement process. Improvements of inversion results could be done with adding in situ hydrographic measurements (like CTD or XBT). Furthermore, the source and receiver geometry and the bottom (and subbottom) properties are exactly know. No track of range and depth will be made because the source and receiver are assumed to remain at a fixed position.

\section{KALMAN FILTERING ALGORITHMS}

The non-linearity (3) in the state-space formulation requires non-linear filtering methods. The most common method is the extended Kalman filter $(\mathrm{EKF})$ that linearizes the process and/or measurement functions around the current state. However, such a linearization can produce unstable filters and degrade the results of the filter [9]. For this reason, new extensions of the Kalman filter have been developed in the last decade. In this paper we empirically compare the performances of EKF with the unscented Kalman filter (UKF) for inverting soundspeed profile in a shallow water environment.

The UKF was first developed by Julier and Uhlmann [9]. Instead of linearizing the dynamics of the system, a statistical transformation approach is developed. The fundamental component of this filter is the unscented transformation. 
The WSSR hypothesis test is based on

$$
\begin{aligned}
& H_{0}: \rho(j) \text { is white } \\
& H_{1}: \rho(j) \text { is not white }
\end{aligned}
$$

Under the null hypothesis, and with a product $L N>30$, $\rho(j)$ can be approximated by a Gaussian $\mathcal{N}(L N, 2 L N)$, so that $95 \%$ of the WSSR statistic must lie below the threshold

$$
\tau=L N+1.96 \sqrt{2 L N}
$$

Filtering algorithms used in this paper come from the REBEL Toolkit [14].

\section{RESULts}

\section{A. Framework}

The Yellow Shark experiments [8], carried out in the Giglio basin of the west coast of Italy during the fall of 1994 (YS94) are used as a realistic case to test the tracking method and evaluate algorithm performance.

The geometric and acoustic parameters used for the following test cases are identical to one of the runs along the main transect of the experiment: the 9-km run. Broadband multitone signal in the frequency band $200-800 \mathrm{~Hz}$ was emitted from a source at $69.2-\mathrm{m}$ depth; the water depth was $113.1 \mathrm{~m}$. The frequencies were $200,250,315,400,500$, 630 and $800 \mathrm{~Hz}$. The transmitted signal was received on a vertical receiving array of 32 hydrophones from $37.2-\mathrm{m}$ to 99.2-m depth with $2-\mathrm{m}$ interelement spacing. The acoustic fields are synthesized using bottom properties measured on sediment cores and through geoacoustic inversion [8], [15]. The bottom is modeled as described on [15] and consists of a 7.5-m thick clay sediment layer with a compression-speed gradient $\left(\rho=1.5 \mathrm{~g} \mathrm{~cm}^{-3}, \mathrm{c}=1470 \mathrm{~m} \mathrm{~s}^{-1}, \mathrm{~g}=2 \mathrm{~s}^{-1}, \alpha=0.03 \mathrm{~dB}\right.$ $\lambda^{-1}$ ) and a homogeneous fluid half space modeling a silty-clay sediment $\left(\rho=1.8 \mathrm{~g} \mathrm{~cm}^{-3}, \mathrm{c}=1530 \mathrm{~m} \mathrm{~s}^{-1}, \alpha=0.15 \mathrm{~dB} \lambda^{-1}\right)$. This environment is depicted in Fig. 2.

The synthesized pressure fields are computed using the normal modes model resolution KRAKEN [16]. Gaussian, zero-mean and uncorrelated measurement noise is added, with a standard deviation of an order of magnitude equal to the half of the amplitude of the pressure fields on the hydrophones.

\section{B. Benchmark results}

The first test case presents a sequence of 20 synthesized profiles. This sequence is constructed with the three first EOF coefficients of the 20 first adjacent SSP from the YS database (9-km run on September 10, 1994).

To ensure a optimal tuning, process covariance used in the filters is equal to the variance of the sequence $P_{x x}=\operatorname{diag}([1.1424 ; 1.2776 ; 0.6021])$. The initial condition of the state is a vector of EOF coefficients close the true values $\left(X_{0}=[-5.0 ; 0.8 ;-0.4]\right.$ in place of $X_{\text {true }}=$ $[-5.44 ; 0.63 ;-0.18])$, with an initial error covariance $P_{0}$ set to $P_{x x}$. So close initial conditions would be obtained by a preliminary inversion on the site, based for example on global optimization methods.

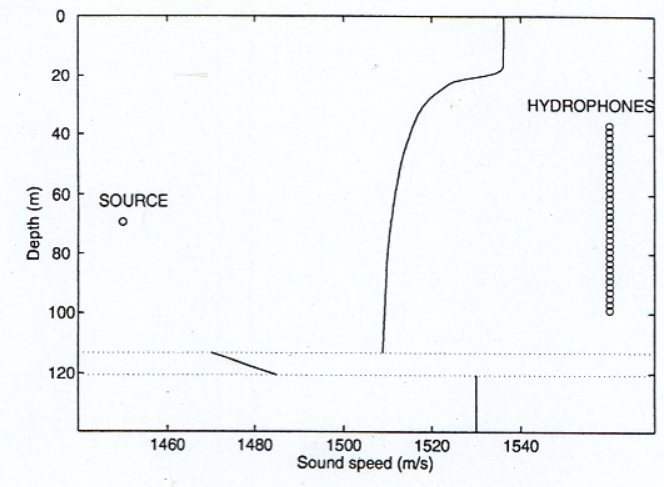

Figure 2. The environment used for the different test cases. The source coordinates are $(0 \mathrm{~km}, 69.2 \mathrm{~m})$ and the 32 hydrophones coordinates are $(5$ $\mathrm{km}, 37.2-99.2 \mathrm{~m})$
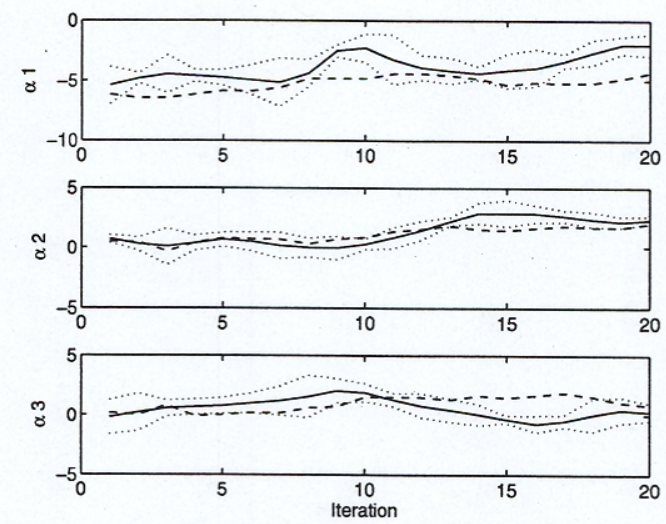

Figure 3. Results of an extended Kalman filter on a sequence of 20 synthesized SSP with 3 EOF coefficients based on YS data set (solid line: true coefficients, dashed line: EKF estimates, dotted line: error bounds $\left.\left( \pm 1.96 \sqrt{P_{x x}}\right)\right)$.

Results of EKF and UKF are shown in Figs. 3 and 4. Variations of the coefficients are better tracked by the UKF processor than by the EKF processor. Indeed, EKF shows more inconsistent results, the estimated EOF coefficients are out of the predicted error bounds on more iterations.

A more interesting illustration of the results quality is the root mean square error of the sound-speed profile $\left(\Delta c_{\mathrm{rms}}\right)$ computed with the filtered states. This parameter is computed as

$$
\Delta c_{\mathrm{rms}}=\sqrt{\frac{1}{D} \sum_{z=z_{1}}^{z_{D}}\left[c_{\mathrm{th}}(z)-c_{\mathrm{filter}}(z)\right]^{2}}
$$

where $D$ is the number of depth points used to discretize the water column.

Figure 5 shows the evolution of this parameter for both the EKF and UKF techniques. The $\Delta c_{\text {rms }}$ rms clearly show better results for the UKF than for EKF, since $80 \%$ of the UKF values are smaller than EKF values. The statistical tests on innovation sequences indicate a better tuning for the UKF processor than for the EKF: $5.36 \%$ of innovation vector components have a 
Like the EKF, the UKF represents the states as Gaussian, but it is specified using a minimal set of deterministically selected samples (the $\sigma$-points). These points capture the mean and covariance of the Gaussian distribution. When they are propagated through the true non-linear process, the posterior mean and covariance are accurately captured to the second order. We give here only the basis principles of the algorithm. More information about algorithmic details and theoretical considerations can be found in [12] and [13].

Consider a state vector of dimension $n$. Unscented transformation will create a collection of $\sigma$-points following :

$$
\begin{aligned}
& \xi_{0}=\bar{x} \\
& \xi_{i}=\bar{x}+\left(\sqrt{(n+\lambda) P_{x x}}\right)_{i} \text { for } i=1, \ldots, n \\
& \xi_{i}=\bar{x}-\left(\sqrt{(n+\lambda) P_{x x}}\right)_{i} \text { for } i=n+1, \ldots, 2 n
\end{aligned}
$$

where $\bar{x}$ and $P_{x x}$ are respectively the mean and the covariance of the state vector and $\lambda$ a scaling parameter that determines the spread of the sigma points.

Each $\sigma$-point is propagated through the prediction process

$$
\boldsymbol{\xi}_{i}\left(t_{k+1} \mid t_{k}\right)=A\left(\boldsymbol{\xi}_{i}\left(t_{k}\right)\right) \text { for } i=0, \ldots, 2 n
$$

and the $a$ priori estimate is computed as

$$
\bar{x}\left(t_{k+1} \mid t_{k}\right)=\sum_{i=0}^{2 n} W_{i} \xi_{i}\left(t_{k+1} \mid t_{k}\right)
$$

with the weights given by

$$
\begin{aligned}
& W_{0}=\frac{\lambda}{n+\lambda} \\
& W_{i}=\frac{\lambda}{2(n+\lambda)} \text { for } i \neq 0
\end{aligned}
$$

The predicted covariance is computed as

$$
\begin{aligned}
P_{x x}\left(t_{k+1} \mid t_{k}\right)= & \sum_{i=0}^{2 n} W_{i}^{c}\left[\xi_{i}\left(t_{k+1} \mid t_{k}\right)-\bar{x}\left(t_{k+1} \mid t_{k}\right)\right] \\
& \times\left[\xi_{i}\left(t_{k+1} \mid t_{k}\right)-\bar{x}\left(t_{k+1} \mid t_{k}\right)\right]^{T}
\end{aligned}
$$

with the weighted terms

$$
\begin{aligned}
& W_{0}^{(c)}=\frac{\lambda}{n+\lambda}+\left(1-\alpha^{2}+\beta\right) \\
& W_{i}^{(c)}=W_{i} \text { for } i \neq 0
\end{aligned}
$$

where $\alpha$ and $\beta$ are the two other scaling parameters.

Each predicted point is used in the measurement model

$$
\Upsilon(k+1 \mid k)=H\left(\boldsymbol{\xi}\left(t_{k+1} \mid t_{k}\right)\right)
$$

and predicted observations are computed as

$$
\boldsymbol{y}\left(t_{k+1} \mid t_{k}\right)=\sum_{i=0}^{2 n} W_{i} \Upsilon_{i}\left(t_{k+1} \mid t_{k}\right)
$$

with covariances

$$
\begin{aligned}
P_{y y}\left(t_{k+1} \mid t_{k}\right)= & \sum_{i=0}^{2 n} W_{i}^{(c)}\left[\Upsilon_{i}\left(t_{k+1} \mid t_{k}\right)-y\left(t_{k+1} \mid t_{k}\right)\right] \\
& \times\left[\Upsilon_{i}\left(t_{k+1} \mid t_{k}\right)-y\left(t_{k+1} \mid t_{k}\right)\right]^{T} \\
& +R_{v v}\left(t_{k+1}\right)
\end{aligned}
$$

Finally, the cross-covariance is computed as

$$
\begin{aligned}
P_{x y}\left(t_{k+1} \mid t_{k}\right)= & \sum_{i=0}^{2 n} W_{i}^{(c)}\left[\boldsymbol{\xi}_{i}\left(t_{k+1} \mid t_{k}\right)-\overline{\boldsymbol{x}}\left(t_{k+1} \mid t_{k}\right)\right] \\
& \times\left[\Upsilon_{i}\left(t_{k+1} \mid t_{k}\right)-\boldsymbol{y}\left(t_{k+1} \mid t_{k}\right)\right]^{T}
\end{aligned}
$$

to obtain the Kalman gain given by

$$
K\left(t_{k+1}\right)=P_{x y}\left(t_{k+1} \mid t_{k}\right) P_{y y}^{-1}\left(t_{k+1} \mid t_{k}\right)
$$

The corrected and predicted states and measurements follow the traditional Kalman filter. In fact, in the exact formulation of the unscented processor, the state vector is augmented with process and measurement noise terms and the process model is rewritten consequently, enabling a generalization of process noise terms in a nonlinear manner.

Besides the parameters $\alpha, \beta$ and $\kappa$, of the UKF, other parameters can influence the performances of the filter: initial values and error covariance of the states and noise terms for process and measurements. A necessary and sufficient condition for filter optimality is that the innovation sequence $e(t)$ (the difference between predicted and measured acoustic pressure fields, on each hydrophone) is zero-mean and uncorrelated [13]. Therefore, the minimum variance filter is considered tuned if and only if this condition is satisfied. If possible, the free parameters will then be adjusted until the condition is achieved.

The zero-mean test will be formulated as follows. Assuming that the processor is Gaussian and ergodic, we test the sample mean $\hat{m}_{e}$ to estimate the population mean $m_{e}$. The sample mean for the $i$ th component of the innovation vector is

$$
\hat{m}_{e}(i)=\frac{1}{N} \sum_{k=1}^{N} e_{i}\left(t_{k}\right) \quad \text { for } \quad i=1, \ldots, L
$$

where $L$ is the size of the observations vector, $N$ the number of iterations and $\hat{m}_{e}(i) \sim \mathcal{N}\left(m_{e}, R_{e e}(i) / N\right)$. At the $5 \%$ significance level (see [13] for more details), the sequence is assumed zero-mean if the sample mean lies below the threshold

$$
\tau_{i}=1.96 \sqrt{\frac{\hat{R}_{e e}(i)}{N}}
$$

where $\hat{R}_{e e}(i)$ is the sample variance estimated by

$$
\hat{R}_{e e}(i)=\frac{1}{N} \sum_{k=1}^{N} e_{i}^{2}\left(t_{k}\right)
$$

The whiteness of the sequence will be tested with the weighted sum squared residual (WSSR). This test aggregates all of the innovation vector information over a finite window of length W (see [13] for more details). The WSSR statistic is defined by

$$
\hat{\rho}(j)=\sum_{k=j-W+1}^{j} e^{T}\left(t_{k}\right) \hat{R}_{e e}^{-1}\left(t_{k}\right) e\left(t_{k}\right) \quad \text { for } \quad j \geq W
$$




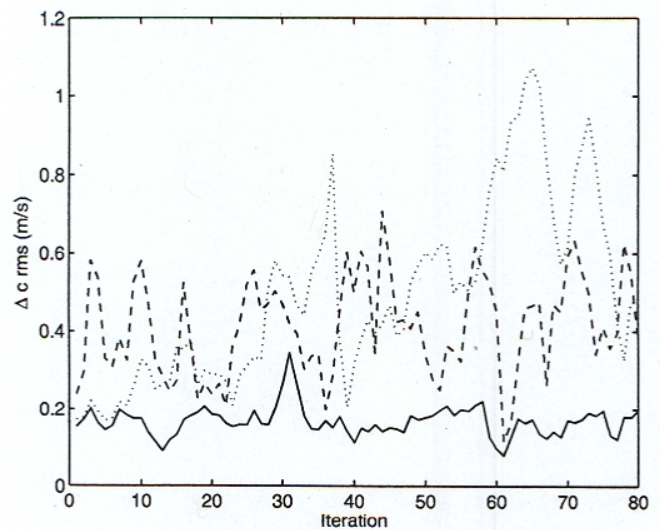

Figure 9. $\Delta c_{\mathrm{rms}}$ for the EKF (dotted line), for the UKF (dashed line) and optimal (solid line). The input data is the entire sequence of real SSP from the YS database. $61 \%$ of $\Delta c_{\text {rms }}$ UKF is smaller than EKF.

\section{CONCLUSION}

Characterize dynamic ocean process and monitor the global states of marine systems is an attractive component where hydroacoustics and oceanographic sciences can interact. Sequential methods are able to update the state of a system with respect to new measurements. For this purpose, use of nonlinear Kalman filter show his potentiality to update the soundspeed profile of a shallow water environment sequentially, by assimilating new broadband acoustic measurements on sparse hydrophone array. Because of the non linearity of our measurement model, the unscented Kalman filter can greatly improve state estimations with respect to the extended Kalman filter. Results are encouraging and use of ensemble method will be the next step of this work. In a parallel direction, inclusion of an oceanographic model, able to theoretically predict global state of the system, will be investigated.

\section{ACKNOWLEDGMENT}

The authors acknowledge the support of the Fonds pour la formation à la Recherche dans l'Industrie et dans l'Agriculture (FRIA), Belgium, and the European Seas Observatory Network Network of Excellence (ESONET NoE) funded by the European 6th Framework Programme, research priority 1.1.6.3 Global change and ecosystems, European Commission.

This work was performed under the auspices of the U.S. Department of Energy by University of California, Lawrence Livermore National Laboratory under Contract W-7405-Eng-48.

\section{REFERENCES}

[1] M. R. Fallat and S. E. Dosso, "Geoacoustic inversion via local, global, and hybrid algorithms," J. Acoust. Soc. Amer., vol. 105, no. 6, pp. 3219 3230, 1999.

[2] J.-P. Hermand, M. Meyer, M. Asch, and M. Berrada, "Adjoint-based acoustic inversion for the physical characterization of a shallow water environment," J. Acoust. Soc. Amer., vol. 119, no. 6, pp. 3860-3871, 2006.

[3] _ _ "Adjoint-based shallow water tomography with partially known bottom properties," in Proceedings of OCEANS '06 Asia Pacific. IEEE, 2006.

[4] J. V. Candy and E. J. Sullivan, "Sound velocity profile estimation: a system theoretic approach," IEEE J. Ocean. Eng., vol. 18, no. 3, pp. 240-252, 1993.

[5] — "Model-based environmental inversion: A shallow water ocean application," J. Acoust. Soc. Amer., vol. 98, no. 3, pp. 1446-1454, 1995.

[6] P. Ellisseeff, H. Schmidt, and W. Xu, "Ocean acoustic tomography as a data assimilation problem," IEEE J. Ocean. Eng., vol. 27, no. 2, pp. 275-282, Apr. 2002.

[7] P. Lermusiaux, "Four-dimensional data assimilation for coupled physical-acoustical fields," Acoustic Variability, 2002, pp. 417-424, 2002.

[8] J.-P. Hermand and P. Gerstoft, "Inversion of broad-band multitone acoustic data from the Yellow Shark summer experiments," IEEE J. Ocean. Eng., vol. 21, no. 4, pp. 324-346, 1996.

[9] S. Julier and J. Uhlmann, "A new extension of the Kalman filter to nonlinear systems," in Int. Symp. Aerospace/Defense Sensing, Simul. and Controls, Orlando, FL, 1997. [Online]. Available: citeseer.ist.psu.edu/julier97new.html

[10] C. Soares, M. Siderius, and S. M. Jesus, "Source localization in a timevarying ocean waveguide," J. Acoust. Soc. Amer, vol. 112, no. 5, pp. 1879-1889, 2002.

[11] P. Gerstoft and D. F. Gingras, "Parameter estimation using multifrequency range-dependent acoustic data in shallow water," J. Acoust. Soc. Amer., vol. 99, no. 5, pp. 2839-2850, 1996.

[12] S. Julier and J. Uhlmann, "A consistent, debiased method for converting between polar and cartesian coordinate systems." [Online]. Available: citeseer.ist.psu.edu/julier97consistent.html

[13] J. V. Candy, Model-Based Signal Processing. New Jersey: John Wiley/IEEE Press, 2006.

[14] R. van der Merwe, "Recursive bayesian estimation library (REBEL) toolkit." [Online]. Available: http://choosh.ece.ogi.edu/rebel/

[15] J.-P. Hermand, "Broad-band geoacoustic inversion in shallow water from waveguide impulse response measurements on a single hydrophone: Theory and experimental results," IEEE J. Ocean. Eng., vol. 24, no. 1, pp. 41-66, 1999.

[16] M. B. Porter, "The KRAKEN normal mode program." [Online]. Available: http://oalib.hlsresearch.com/Modes/kraken.pdf 

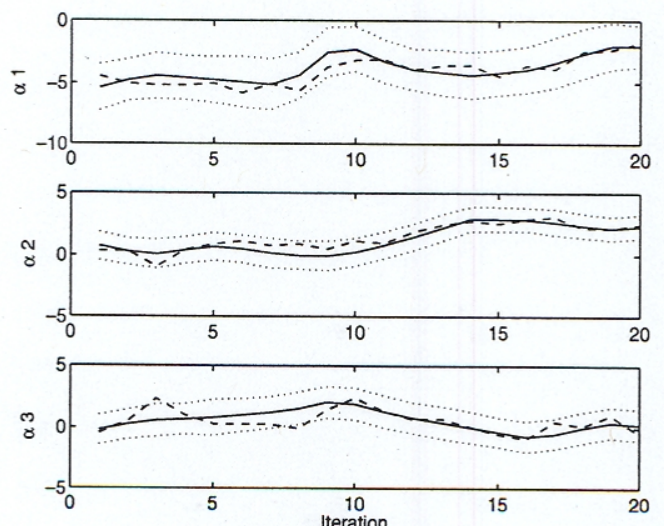

Figure 4. Results of an unscented Kalman filter on a sequence of 20 synthesized SSP with 3 EOF coefficients based on YS data set (solid line: true coefficients, dashed line: UKF estimates, dotted line: error bounds $\left.\left( \pm 1.96 \sqrt{P_{x x}}\right)\right)$.

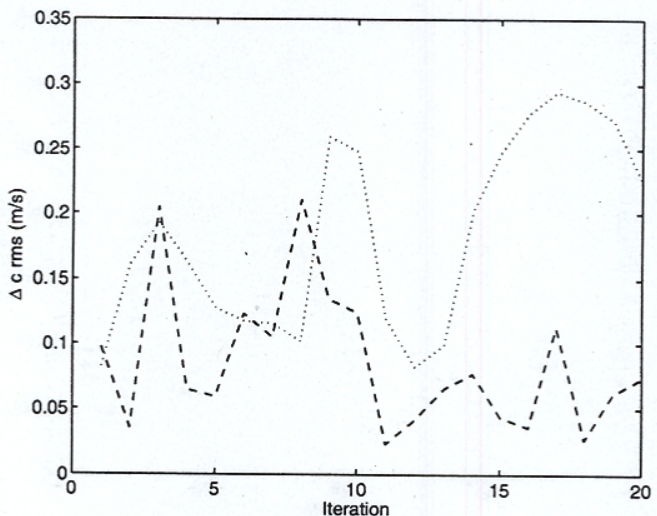

Figure 5. $\Delta c_{\text {rms }}$ for the EKF (dotted line) and for the UKF (dashed line). The input SSP is a sequence of 20 synthesized SSP.

mean above their threshold for the EKF, for only $1.33 \%$ for the UKF. The WSSR statistical tests give $9.10 \%$ out for the EKF and $0 \%$ out for the UKF (Fig. 6).

\section{Real data sequence}

The second test is a run on the entire YS sequence. This sequence of profiles is shown in Fig. 7 in the region of 10- to 40-m water depths.

Results of both filters are presented in Fig. 8. The statespace model is clearly not optimal to describe the SSP evolution, since the transition matrix is the identity. However, the $\mathrm{UKF}$ tracks reasonably well the EOF coefficients and performs better than EKF (in the mean of $\Delta c_{\mathrm{rms}}$ ) for $61 \%$ of the sequence.

Figure 9 shows the $\Delta c_{\text {rms }}$ for the two filters. The third (solid) line is the minimum that is possible to obtain, with the sequence of true EOF coefficients. We use a finite number of EOF, so that the reconstruction of the SSP cannot match exactly with the real SSP used to synthesize pressure field.

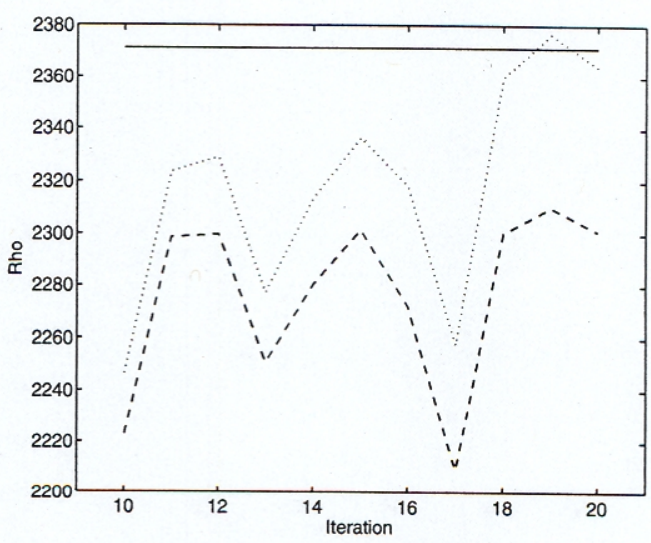

Figure 6. WSSR test for the EKF (dotted line, $9.10 \%$ out) and for the UKF (dashed line, $0 \%$ out). The solid line is the WSSR threshold (19). The input SSP is a sequence of 20 synthesized SSP.

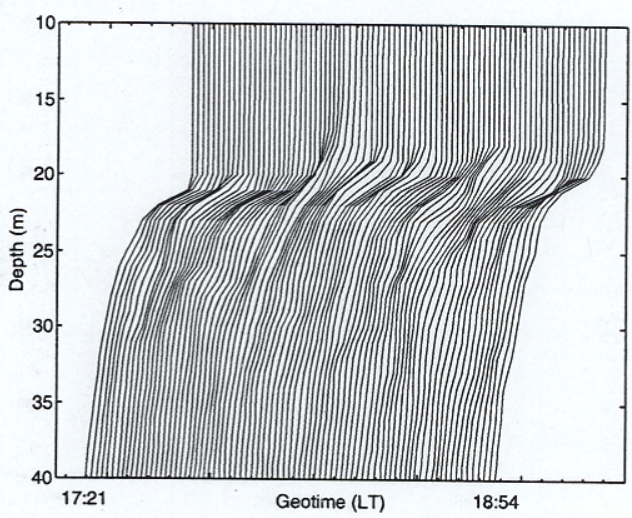

Figure 7. Zoom on the entire sequence of the 80 sound-speed profiles from the YS database. Region of 10 - to $40-\mathrm{m}$ depth. One minor tickmark corresponds to $4 \mathrm{~m} / \mathrm{s}$.
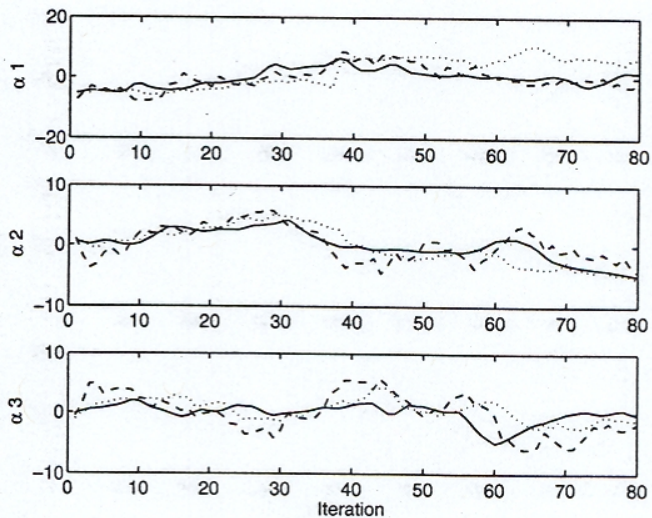

Figure 8. State estimations of EKF (dotted line), UKF (dashed line) and true coefficients (solid line). The input data is the entire sequence of real SSP from the YS database. 